\title{
Solid-state NMR enhanced by dynamic nuclear polarization as a novel tool for ribosome structural biology
}

\author{
Ioannis Gelis • Veronika Vitzthum • Neha Dhimole • \\ Marc A. Caporini - Andreas Schedlbauer - Diego Carnevale • \\ Sean R. Connell · Paola Fucini · Geoffrey Bodenhausen
}

Received: 29 November 2012 / Accepted: 7 March 2013/Published online: 21 May 2013

(C) Springer Science+Business Media Dordrecht 2013

\begin{abstract}
The impact of Nuclear Magnetic Resonance (NMR) on studies of large macromolecular complexes hinges on improvements in sensitivity and resolution. Dynamic nuclear polarization (DNP) in the solid state can offer improved sensitivity, provided sample preparation is optimized to preserve spectral resolution. For a few nanomoles of intact ribosomes and an $800 \mathrm{kDa}$ ribosomal complex we demonstrate that the combination of DNP and magic-angle spinning NMR (MAS-NMR) allows one to overcome current sensitivity limitations so that homo- and heteronuclear ${ }^{13} \mathrm{C}$ and ${ }^{15} \mathrm{~N}$ NMR correlation spectra can be recorded. Ribosome particles, directly pelleted and frozen
\end{abstract}

Electronic supplementary material The online version of this article (doi:10.1007/s10858-013-9721-2) contains supplementary material, which is available to authorized users.

I. Gelis · N. Dhimole - A. Schedlbauer .

S. R. Connell · P. Fucini

Buchmann Institute for Molecular Life Sciences, Institute of Organic Chemistry and Chemical Biology, Goethe University Frankfurt, Max-von-Laue-Str. 15, 60438 Frankfurt am Main, Germany

Present Address:

I. Gelis

Department of Chemistry, University of South Florida, 4202

East Fowler Ave, CHE 205, Tampa, FL 33620, USA

V. Vitzthum - M. A. Caporini - D. Carnevale

G. Bodenhausen $(\bowtie)$

Institut des Sciences et Ingénierie Chimiques, Ecole

Polytechnique Fédérale de Lausanne, 1015 Lausanne,

Switzerland

e-mail: geoffrey.bodenhausen@epfl.ch

A. Schedlbauer · S. R. Connell · P. Fucini $(\square)$

Structural Biology Unit, CIC bioGUNE, Bizkaia Technology

Park, 48160 Derio, Spain

e-mail: pfucini@cicbiogune.es into an NMR rotor, yield DNP signal enhancements on the order of $\sim 25$-fold and spectra that exhibit narrow linewidths, suitable for obtaining site-specific information. We anticipate that the same approach is applicable to other high molecular weight complexes.

Keywords Ribosome structure $\cdot$ Magic-angle spinning NMR (MAS-NMR) · Solid-state NMR · Dynamic nuclear polarization (DNP) · TOTAPOL

\section{Introduction}

Notwithstanding formidable advances in structural biology, the study of large macromolecular complexes, ranging from hundreds of kilodaltons to several megadaltons,

S. R. Connell · P. Fucini

IKERBASQUE, Basque Foundation for Science, 48011 Bilbao, Spain

G. Bodenhausen

Département de Chimie, Ecole Normale Supérieure, 24 rue

Lhomond, 75231 Paris Cedex 05, France

G. Bodenhausen

Université Pierre-et-Marie Curie, Paris, France

G. Bodenhausen

Laboratoire des Biomolécules, UMR 7203, CNRS/UPMC/ENS,

Paris, France 
remains a difficult task. The challenges associated with understanding the ribosome structure and dynamics in its many functional states are representative of the strengths and shortcomings of today's approaches to structurally characterize large cellular complexes. The ribosome is a macromolecular machine responsible for cellular protein synthesis that, even in simple organisms such as Escherichia coli, reaches the size of more than $2.3 \mathrm{MDa}$. Determining the structure of the ribosome has been the aim of numerous investigations since the early 1980's. These studies have recently culminated in the accurate description of the ribosomal architecture, thus shedding light on its role in the basic steps of translation including the two most fundamental aspects of protein synthesis, peptide bond formation and decoding (Noeske and Cate 2012; Schmeing and Ramakrishnan 2009), as well as describing its interaction with major classes of antibiotics (Wilson 2009).

These results set the stage for studying evermore interesting functional complexes, where one of the most ambitious goals is to visualize at the atomic level how the interplay between the ribosome, protein folding and sorting machinery guide the newly synthetized polypeptide nascent chain toward its transformation into a functional protein. Our progress, in this respect, resides not only in the type of samples that we can prepare but in the type of sample that we can indeed analyze. Thus far this analysis predominantly relied on X-ray crystallography and cryoelectron microscopy (cryo-EM). These methods are best suited to complexes with high stability, high ligand occupancy, low conformational heterogeneity, and in the case of X-ray diffraction, with the propensity to form crystals that diffract at high resolution. However our advancement in ribosome structural biology hinges on the analysis of functional processes that do not meet these criteria, forcing us to look for alternative approaches.

Complementing biochemical and cryo-EM studies, solution-state NMR has been the only technique able to provide high-resolution structural information on one of the most intriguing and insufficiently understood phenomena of structural biology, non-assisted co-translational protein folding (Fedyukina and Cavagnero 2011). By building on initial studies characterizing the highly dynamic and functionally essential L7/L12 ribosomal stalk (Christodoulou et al. 2004; Mulder et al. 2004), the way was paved to follow the structure and dynamics of long polypeptide nascent chains retained on stalled ribosomal complexes (Hsu et al. 2007, 2009; Cabrita et al. 2009; Rutkowska et al. 2009; Eichmann et al. 2010). The achievement of acquiring solution-state NMR spectra from a ribosomal complex of more than $2.3 \mathrm{MDa}$, is made possible by the inherent high-flexibility of L7/L12 and the polypeptide nascent chain. In both cases, the region of the protein that is analyzed is linked to the ribosome by a short and unstructured stretch of 10-20 amino acids. Local motions of these regions shorten the effective correlation times so that the ${ }^{1} \mathrm{H}$ linewidths of the first $100 \mathrm{~N}$-terminal amino acids of a 150 residues long nascent chain tethered to the ribosome at its $\mathrm{C}$-terminus are comparable to those of the same protein in its untethered form (Hsu et al. 2007). Similarly, the short correlation time observed for the L7/ L12 stalk ( $\left.\tau_{\mathrm{c}}=15 \mathrm{~ns}\right)$ suggests that this region tumbles, to a large extent, independently of the ribosomal body (Christodoulou et al. 2004). Solution-state NMR has also been successfully applied to study the dynamic binding of different translation factors such as IF-1, EF-G, EF-Tu, IF-2 and RF3 (Sette et al. 1997; Christodoulou et al. 2004; Helgstrand et al. 2007). Although the large molecular weight of a ribosome implies very slow rotational diffusion $\left(\tau_{\mathrm{c}} \sim 2.5 \mu \mathrm{s}\right.$ for E. coli ribosome at $20^{\circ} \mathrm{C}$ ) (Amand et al. 1977 ) and, therefore, very broad non-detectable lines for any element tightly associated with the ribosome, indirect information about the bound state of the factor can be retrieved by inspecting changes in the resonances of the free state. When for example IF1 interacts with the ribosome, it is in fast chemical exchange, i.e., the on- and offrates are faster than the difference between the chemical shifts of the bound and free states. This allows one to identify residues of IF1 involved in binding to the ribosome (Sette et al. 1997) via changes of their averaged chemical shifts and line-widths.

Despite the demonstrated ability of solution-state NMR to study the structure of dynamic regions of ribosome complexes that are poorly represented or 'invisible' in electron density maps, this approach also has short-comings. To avoid aggregation, ribosome concentrations must be limited to $\sim 30 \mu \mathrm{M}$. Even at such low concentrations, the samples tend to degrade at $25{ }^{\circ} \mathrm{C}$ after $\sim 24 \mathrm{~h}$, so extensive time-averaging must be ruled out. These limitations strongly restrict the type of NMR experiments and hence structural information that can be obtained. Indeed most of our knowledge has derived from the comparison of HSQC and HMQC spectra. It remains an unsolved task to use solution-state NMR to solve 'de-novo' the structure of ribosome elements or ligands, in their bound state.

To circumvent these limitations, our attention has been drawn by the recent advances reported in the solid state NMR field.

Solid-state NMR using magic-angle spinning (MASNMR) is emerging as a popular technique for structural biology, since it allows studying macromolecular complexes that are too large for solution-state NMR, yet cannot be studied by electron diffraction techniques (Ader et al. 2010; Cady et al. 2010; Hiller et al. 2005; Shewmaker et al. 2006; Wasmer et al. 2008). When combined with DNP (Abragam 1961; Abragam and Goldman 1978) the sensitivity can be improved to the extent that high-quality $2 \mathrm{D}$ 
correlation maps of nanomolar quantities of protein complexes can be obtained in reasonable experimental times (Reggie et al. 2011). The DNP enhancement can be achieved by the addition of stable radicals such as TOTAPOL to the solvent (Song et al. 2006), by covalent grafting of such radicals to the proteins (Vitzthum et al. 2011), and by saturating the EPR transitions with a powerful microwave source such as a gyrotron (Hall et al. 1997). Sensitivity enhancements on the order of $\varepsilon_{\text {on/off }}$ $\sim 200$ (i.e., the ratio of signal intensities obtained with and without microwave irradiation) have been observed in frozen glycerol/water matrices at temperatures in the vicinity of $100 \mathrm{~K}$ (Kiesewetter et al. 2012), resulting in a reduction of experimental times by a factor of $\left(\varepsilon_{\mathrm{on} / \mathrm{off}}\right)^{2}=$ 40,000 allowing this approach to be applied to a variety of molecular systems beyond the reach of traditional methods. For example, applications to surface science (Vitzthum et al. 2012; Zagdoun et al. 2012; Lelli et al. 2011) have been remarkably successful and may open the way to improved understanding of heterogeneous catalysis and other phenomena that can only occur on surfaces. DNPenhanced MAS-NMR has so far been applied to study the structure of only few macromolecules, fibrils and protein aggregates (Debelouchina et al. 2010; Bayro et al. 2011), membrane proteins (Reggie et al. 2011; Bajaj et al. 2009) and whole cells (Renault et al. 2012).

In this communication, we report our strategies and initial experiments to adapt DNP-enhanced MAS-NMR to study ribosome complexes. We demonstrate that using a newly designed home-built centrifugal device ribosomal complexes can be pelleted and concentrated by ultra-centrifugation directly in NMR rotors. The sample analyzed yields signal enhancements on the order of $\varepsilon_{\text {on/off }} \sim 25$ at temperatures near $100 \mathrm{~K}$, overcoming the problems of ribosomal complex stability. The spectra feature linewidths on the order of $1 \mathrm{ppm}$, or $100 \mathrm{~Hz}$ for carbon-13 signals at $\mathrm{B}_{0}=9.4 \mathrm{~T}\left({ }^{1} \mathrm{H}\right.$ and ${ }^{13} \mathrm{C}$ Larmor frequencies of 400 and $100 \mathrm{MHz}$ respectively) allowing one to obtain information at atomic resolution, particularly when multidimensional NMR is combined with selective isotope labeling.

\section{Results and discussion}

Design of centrifugal devices for ribosome packing

Although DNP-enhanced MAS-NMR has major advantages for biological systems, its sensitivity is relatively low. The simplest way to increase the sensitivity is to increase the concentration of the material packed in the NMR rotor. Sample manipulation and rotor packing are critical steps for DNP-enhanced MAS-NMR. We developed custom- designed concentrators for direct pelleting of intact $E$. coli $70 \mathrm{~S}$ ribosomes (E70S) and ribosome subunits into an NMR rotor by ultracentrifugation. The devices described in the Supporting Information (Figure S1) are designed to fill $3.2 \mathrm{~mm}$ NMR rotors using Beckman ultracentrifuges with either SW28/SW32 swinging-bucket or Ti-45 fixed-angle rotors. In contrast to similar tools developed for MASNMR studies of biomolecules by ultracentrifugation (Gardiennet et al. 2012; Bertini et al. 2012a) or by direct packing in an NMR rotor (Bertini et al. 2011; 2012b) the devices presented here have a large reservoir $(6-7 \mathrm{ml})$, allowing ratios between the reservoir and the rotor volumes of $\sim 230$ and $\sim 200$. These systems are, therefore, suitable for pelleting proteins over a wide range of concentrations and are particularly attractive for packing macromolecular complexes that are prone to aggregation. There are several practical advantages associated with direct packing into an NMR rotor: (1) only a single pelleting step is required, (2) minimal sample loss during the transfer of costly preparations, (3) no need for preparing micro- or nanocrystalline samples by precipitation with PEG or other auxiliaries/ precipitants, and (4) the resulting pellets contain a large amount of buffer, so that the macromolecular complex under investigation remains in a close-to-native condition.

Frozen ribosomal pellets are suitable for DNPenhanced MAS-NMR

To draw benefits from DNP-enhanced MAS-NMR and obtain large and uniform enhancements, the solute must be dissolved in a solvent mixture that forms a homogeneous glassy matrix that facilitates spin diffusion at cryogenic temperatures. At the same time, the matrix acts as a cryoprotectant for biomolecular complexes (Hall et al. 1997). To assess whether frozen ribosomal pellets containing some residual buffer are useful for DNP studies, we explored the effect of different solvents and TOTAPOL concentrations on $\varepsilon_{\text {on/off }}$ by recording one-dimensional ${ }^{13} \mathrm{C}$ spectra of E70S particles, at natural isotopic abundance, obtained by cross-polarization (CP) from protons with or without microwave irradiation at $100 \mathrm{~K}$ and $9.4 \mathrm{~T}$ (400 MHz for protons, $263 \mathrm{GHz}$ for electrons). The E70S particles were pelleted from a buffer of glycerolD8: $\mathrm{D}_{2} \mathrm{O}: \mathrm{H}_{2} \mathrm{O}$ (60:30:10), with $20 \mathrm{mM}$ TOTAPOL. The spectra (Fig. 1) reveal an enhancement factor $\varepsilon_{\text {on/off }} \sim 25$ for ribosome signals. The glycerol signals near $70 \mathrm{ppm}$ exhibit the same $\varepsilon_{\text {on/off }}$ ratio, suggesting that the packed E70S particles retain a sufficient amount of solvent for the dipolar couplings between the free electrons of the biradical and the protons of the solute to be effective (Debelouchina et al. 2010; van der Wel et al. 2006). Increasing the biradical concentration to $40 \mathrm{mM}$ reduces the longitudinal relaxation times and allows one to choose a shorter 


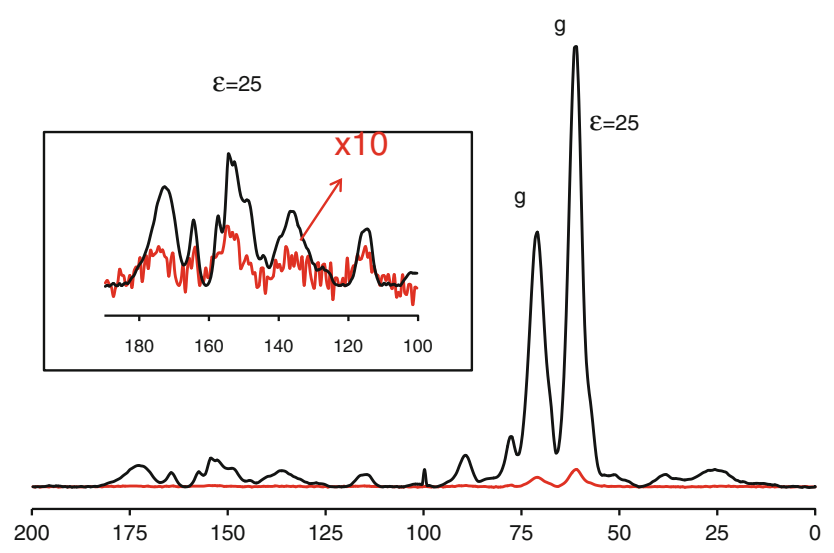

Fig. 1 Carbon-13 spectra of a frozen pellet of unlabeled E70S (3.5 nmoles in $30 \mu \mathrm{l}$ ) acquire in $40 \mathrm{~min}$ by cross-polarization (CP) from protons to ${ }^{13} \mathrm{C}$ (contact time $2.4 \mathrm{~ms}$ ) at $9.4 \mathrm{~T}$ ( $400 \mathrm{MHz}$ for protons), $\mathrm{T}=100 \mathrm{~K}$, MAS spinning frequency of $v_{\text {rot }}=8 \mathrm{kHz}, 256$ scans with a relaxation delay of $9.4 \mathrm{~s}$. E70S was pelleted from a HEPES buffer $(\mathrm{pH}=7.6)$ in 60:30:10 D8-glycerol: $\mathrm{D}_{2} \mathrm{O}: \mathrm{H}_{2} \mathrm{O}$, containing $20 \mathrm{mM}$ TOTAPOL. A DNP enhancement $\varepsilon_{\text {on/off }}=25$ was determined for both E30S-IF1 and glycerol signals (denoted by $g$ ) by acquiring spectra with the $263 \mathrm{GHz}$ microwave irradiation 'on' (black) or 'off' (red)

relaxation delay and a faster repetition rate. However, this gain is not accompanied by a higher $\varepsilon_{\text {on/off }}$ for the E70S signals (only glycerol signals exhibit slightly higher $\varepsilon_{\text {on/off }}$, as shown in Figure S2) or higher signal-to-noise ratio per unit time, presumably due to losses in signal intensity arising from the paramagnetic effects of the biradical (Lange et al. 2012). Since sucrose is extensively used in ribosome preparations and aqueous sugar solutions are known to be very effective in promoting the formation of amorphous ice at low temperatures (Angell 2002), we tested whether glycerol can be replaced by an aqueous sucrose solution (Figure S2). Intriguingly, the DNP enhancement obtained when E70S is pelleted from a $30 \%$ (w/v) sucrose DNP buffer, in $\mathrm{D}_{2} \mathrm{O}: \mathrm{H}_{2} \mathrm{O}$ (90:10), with $20 \mathrm{mM}$ TOTAPOL was only $\varepsilon_{\text {on/off }} \sim 13$, significantly lower than for glycerol. We assume that this reduction in $\varepsilon_{\text {on/off }}$ is due to a less homogeneous distribution of the biradicals upon freezing, which depends on both buffer composition and rate of freezing (Barnes et al. 2008; Georgieva et al. 2012). Nevertheless, the higher E70S packing density achieved when pelleting with sucrose ( $5.5 \mathrm{nmol} /$ rotor for sucrose vs. $3.5 \mathrm{nmol} /$ rotor for glycerol) partially compensates for the lower $\varepsilon_{\text {on/off }}$ and results in a satisfactory signal-to-noise ratio ( $\sim 20$ for the carbonyl region). Therefore, sucrose can be a very attractive glassforming medium for DNP-NMR studies of biological macromolecules that are purified by centrifugation through sucrose gradients or cushions.

The highest DNP-enhancement we observed $\left(\varepsilon_{\text {on/off }}\right.$ $\sim 80$ ) was obtained with a frozen solution of IF1 (Figure S3) at $100 \mathrm{~K}$ in buffer containing $60 \%$ glycerol and
$20 \mathrm{mM}$ TOTAPOL (see below). This large $\varepsilon_{\mathrm{on} / \mathrm{off}}$ is due in part to bleaching which appears to be more effective in a frozen solution than in a pellet that contains only small amounts of solvent.

High-resolution DNP-enhanced MAS NMR reveals contact residues in an $800 \mathrm{kDa}$ complex

The enhancement factors obtained for unlabeled E70S ribosomes prompted us to record homo- and heteronuclear 2D correlation spectra on labeled ribosome complexes of the Escherichia coli small ribosomal subunit (E30S) and IF1. The relatively high affinity of IF1 for E30S (Celano et al. 1988) makes it an ideal system for this study since it allows a high concentration of the E30S-IF1 complex in the NMR rotor with only small amounts of free E30S (see Supplementary Information). In addition, this complex is representative for a broad class of ribosome complexes, where a factor bound to the surface of a ribosomal subunit is exposed to the solvent that contains the radicals (Fig. 2a). Even more importantly, the mode of binding of IF to E30S has already been characterized, both by solution-state NMR (Sette et al. 1997) as well as by X-ray crystallography (Carter et al. 2001), providing the framework for comparing and validating our results.

The DNP-enhanced proton-driven spin-diffusion (PDSD) ${ }^{13} \mathrm{C}-{ }^{13} \mathrm{C}$ correlation spectrum of an E30S-IF1 complex, where only IF1 is uniformly labeled with ${ }^{13} \mathrm{C}$ and ${ }^{15} \mathrm{~N}$ (Fig. 3), shows a very good signal-to-noise ratio $\left(\varepsilon_{\mathrm{on} / \mathrm{off}}\right.$ $\sim 25$ ), although the amount of sample is only $\sim 30 \mathrm{nmol} /$ rotor (with an estimated $90 \%$ occupancy of IF1 on the ribosome and less than $0.01 \%$ of free IF1, Supplementary Information). However, the linewidths lead to signal overlap even in regions where only a few signals are expected. This spectral crowding is attributed to inhomogeneous broadening caused by the conformational heterogeneity of E30S-IF1 in the frozen wet pellet, possibly compounded by abrupt freezing when the rotor is suddenly immersed in liquid nitrogen (Linden et al. 2011; Martin and Zilm 2003). To alleviate crowding and improve spectral resolution, we selectively labeled the tyrosine and histidine residues present in IF1 with ${ }^{13} \mathrm{C}$ and ${ }^{15} \mathrm{~N}$. As expected from the sequence of IF1, the PDSD spectrum (Fig. 3) reveals a set of five signals in the dispersed CO-CA region, whereas the $\mathrm{CA}-\mathrm{CB}$ region is masked by the signals of glycerol. Selective labeling of the two Tyr residues further simplifies the spectrum, resulting in two completely resolved signals. This allows an unambiguous distinction between the two types of amino acids (Figure S5). The linewidths of the two Tyr signals in the direct dimension are 100 and $120 \mathrm{~Hz}$ (1.0 and $1.2 \mathrm{ppm}$ ), much like what has been observed for the membrane protein bacteriorhodopsin embedded in its native lipid bilayer (Barnes et al. 2010) at a 


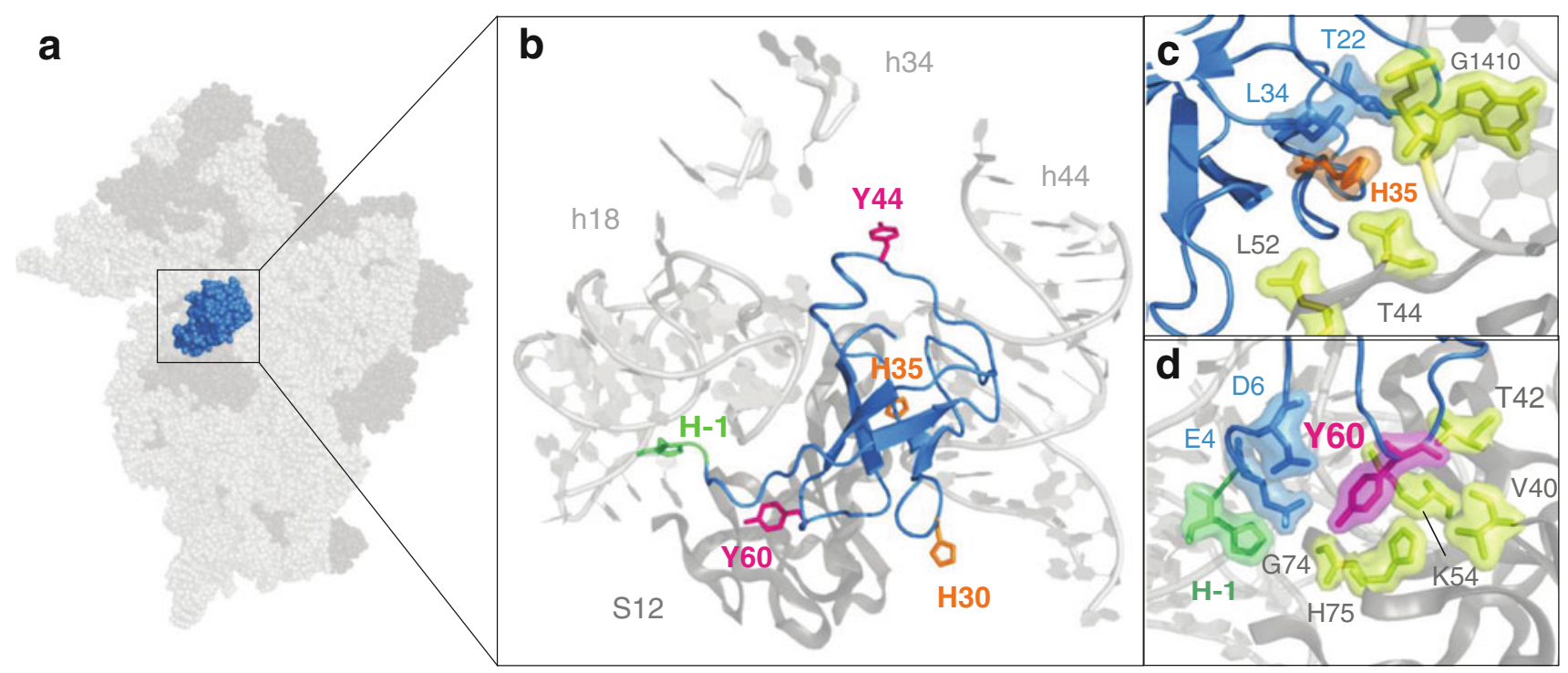

Fig. 2 Interaction between IF1 and the small ribosome subunit, the E30S-IF1 complex. a Overview of the complex, as reported in the X-ray crystallography study (PDB ID: 1HR0, Carter et al. 2001), between $T$. thermophilus 30S (light and dark gray for the ribosomal RNA and proteins, respectively) and T. thermophilus IF1 (marine blue). b Closed-up view of the interaction site, i.e. boxed region in panel (a), which involves the rRNA helices h18 and h44 (light gray) and the ribosomal protein S12 (dark gray). Using the high structural similarity between the E. coli and T. thermophilus IF1 structures in their free and bound states (PDB IDs: 1HR0 and 1AH9, Figure S4), the positions of the residues labeled in this study are indicated such that His 30 and His35 are orange, and Tyr44 and Tyr60 are magenta. The additional Histidine residue present in the IF1 protein used in this study in position -1 (His -1$)$ is colored green. Of the five labeled residues, His -1 , His 35 and Tyr60 are in close contact with the ribosome while His30 and Tyr44 are exposed to the solvent. c The environment around residue His35 (orange surface) of IF1 (marine blue ribbon) is shown. The elements found in the crystal structure at a distance less than $5 \AA$ from His35 are indicated in a surface representation. These include residues from the ribosomal protein S12 or rRNA (yellow) as well as IF1 residues (light blue). d The binding pocket of Tyr60 (magenta surface) of IF1 (marine blue ribbon) is shown where elements found in the crystal structure at a distance less than $5 \AA$ from Tyr60 are indicated in a surface representation and colored as in panel (c). In addition the modeled position of His-1 is shown and colored green similar temperature. These linewidths suggest that neither the cryogenic temperatures nor the state of the frozen pellets are limiting factors in obtaining well-resolved spectra. Indeed, even for nano-crystalline protein preparations, resonance linewidths are dominated by inhomogeneous broadening at cryogenic temperatures (Linden et al. 2011). A direct comparison between the PDSD ${ }^{13} \mathrm{C}-{ }^{13} \mathrm{C}$ spectrum of His,Tyr-labeled IF1 in complex with E30S (Fig. 4, red lines) and a spectrum of free IF1 (Fig. 4, blue contours), acquired under identical conditions (at $100 \mathrm{~K}$ in a DNP-buffer with $60 \%$ glycerol and $20 \mathrm{mM}$ TOTAPOL) yields useful insight into the residues that are involved in the E30S-IF1 interaction. The $\mathrm{H}_{\text {II }}$ and $\mathrm{H}_{\text {III }}$ resonances observed for the IF1-E30S complex (Fig. 4, red spectrum), are absent in the spectrum acquired with IF1 in its free state (Fig. 4, blue spectrum). Although it is impossible to determine whether $\mathrm{H}_{\mathrm{II}}$ and $\mathrm{H}_{\mathrm{III}}$ experience a large change in chemical shift upon E30S binding, or whether their resonances are broadened beyond detection in the free state (due to either paramagnetic broadening or the "freezing" of multiple conformations), their different appearance in the two spectra indicates that these two residues are clearly affected by the presence of the ribosome. A similar reasoning can be applied to $\mathrm{Y}_{\mathrm{I}}$ although the observed change is less significant when the two spectra are compared. On the contrary, the resonances for $\mathrm{H}_{\mathrm{I}}$ and $\mathrm{Y}_{\mathrm{II}}$ are identical in the two spectra, indicating that the physical and chemical environment of these two residues remains the same upon binding to the ribosome.

The available X-ray crystallography and solution-state NMR structures of the IF1-30S complex (Carter et al. 2001; Sette et al. 1997) and unbound IF1 (Sette et al. 1997), allow us to compare, validate and further interpret the results we obtained using DNP enhanced MAS-NMR. When comparing IF1 in its free or bound state as reported respectively by solution-state NMR (Sette et al. 1997) and X-ray crystallography (Carter et al. 2001), it is evident that IF1 does not undergo a substantial conformational change upon binding to the $30 \mathrm{~S}$ (Fig S4), in a groove formed between ribosomal protein S12 and the rRNA helices 44 and 18 (h44, h18) (Fig. 2b). A closer inspection of the IF1$30 \mathrm{~S}$ structure reveals that of the 5 IF1 residues labeled in our DNP-NMR experiments, only His30 and Tyr44 are not involved in binding (Fig. 2b). His-1, His35 and Tyr60, on 


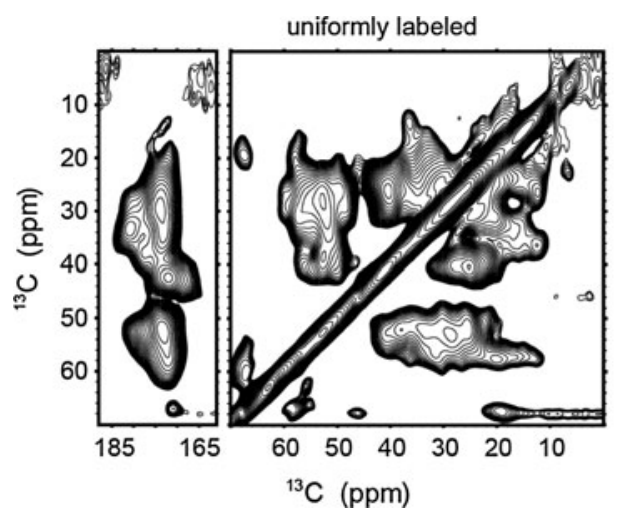

Fig. 3 Proton-driven spin diffusion (PDSD, $\tau_{\text {mix }}=18 \mathrm{~ms}$ ) ${ }^{13} \mathrm{C}-{ }^{13} \mathrm{C}$ correlation spectrum of frozen pellets of uniformly ${ }^{13} \mathrm{C}$-enriched IF1 in complex with E30S obtained with cross-polarization (CP) at $9.4 \mathrm{~T}$, $\mathrm{T}=100 \mathrm{~K}$ and $v_{\text {rot }}=8 \mathrm{kHz}$. Cryogenic temperatures inhibit the averaging of chemical shifts by internal motions in the amorphous

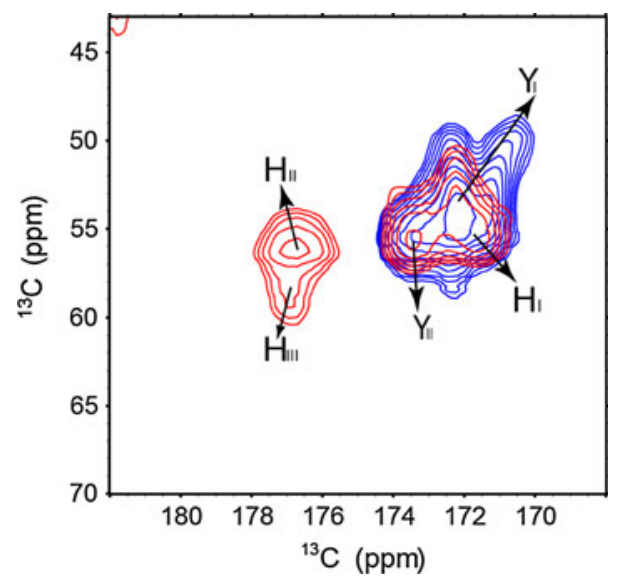

Fig. 4 Overlay of a ${ }^{13} \mathrm{C}-{ }^{13} \mathrm{C}$ PDSD correlation spectrum (CO-CA region) of free (blue) and E30S-bound (red) His,Tyr-labeled IF1. The spectrum of the free form suffers from extensive overlap due to degenerate chemical shifts. Selective labeling of Tyr or both Tyr and His residues in IF1 (see Fig. 2) allows differentiating between the two types of amino acids in the bound form. Comparison between the free and bound states suggests that two of the His residues are strongly affected by binding $\left(\mathrm{H}_{\mathrm{II}}\right.$ and $\left.\mathrm{H}_{\mathrm{III}}\right)$, while of the two Tyr residues only one is not affected $\left(\mathrm{Y}_{\mathrm{II}}\right)$

the contrary, are deeply buried in the interaction interface (Fig. 2b-d). These observations provide the basis for a tentative assignment of the resonances observed in our spectra. $\mathrm{H}_{\text {II }}$ and $\mathrm{H}_{\text {III }}$, the two His resonances (Fig. 3, central and right panels) that experience the strongest changes upon binding to the ribosome (Fig. 4) are thus likely to be generated by His -1 and His 35 , the two His residues that are in close contact with the ribosome in the X-ray structure (Fig. 2b-d). Moreover, $\mathrm{H}_{\mathrm{I}}$ can be assigned to His30 as this resonance is not altered when the spectra of the free to the bound state are compared (Fig. 4) and, as seen in the crystal structure, H30 does not interact with the ribosome, so that its chemical and physical environment are the same

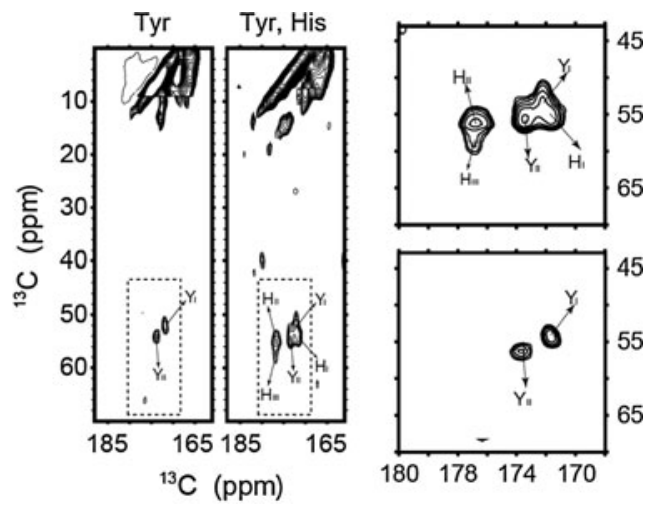

sample and lead to signal broadening and extensive overlap in both aliphatic and CO-CA regions (left panel). Selective labelling of only Tyr residues, or of both Tyr and His residues, overcomes this limitation and yields almost completely resolved signals (central panels). Dashed boxes are magnified on the right

in its free or bound form (Fig. 2b). Following a similar reasoning $\mathrm{Y}_{\mathrm{II}}$ can be assigned to $\mathrm{Y} 44$ for which no significant interaction with the ribosome are observed in the $\mathrm{X}$-ray structure of the complex (Fig. 2b), implying no changes in its chemical environment upon binding to the ribosome and accordingly no chemical shift changes are observed for this residue in the comparison of the free IF1 versus IF1-E30S spectrum (Fig. 4). Finally, the last labeled residue, $\mathrm{Y} 59$, can be assigned to $\mathrm{Y}_{\mathrm{I}}$. It is worth mentioning that, although we do observe chemical shift changes between the free and bound forms in the aromatic region (Figure S6), the lack of aromatic-aliphatic correlations does not allow one to assign these resonances to specific residues.

DNP allows detecting $2 \mathrm{D}{ }^{15} \mathrm{~N}-{ }^{13} \mathrm{C}$ correlations and spin-system identification

To assess the extent to which the heterogeneity of the E30S-IF1 pellets affects the spectra used for identification of spin systems and for sequential assignments, we recorded a NCACX experiment, which employs two consecutive cross-polarization steps, initially from ${ }^{1} \mathrm{H}$ to ${ }^{15} \mathrm{~N}$, followed by transfer from ${ }^{15} \mathrm{~N}$ to ${ }^{13} \mathrm{C}$ (Baldus et al. 1998; Pauli et al. 2001; Egorova-Zachernyuk et al. 2001). Like the PDSD ${ }^{13} \mathrm{C}-{ }^{13} \mathrm{C}$ spectrum of uniformly labeled ${ }^{13} \mathrm{C}$, the central $\mathrm{N}_{\mathrm{i}} \mathrm{CA}_{\mathrm{i}}, \mathrm{N}_{\mathrm{i}} \mathrm{CB}_{\mathrm{i}}$ and $\mathrm{N}_{\mathrm{i}} \mathrm{CO}_{\mathrm{i}-1}$ regions of the NCACX spectrum collected from a ${ }^{15} \mathrm{~N}$-labeled IF1 in complex with E30S suffer from signal overlap (Figure S7). Nevertheless, several signals can be resolved, including three distinct spin systems that belong to Thr and Ala residues (Fig. 5). Overall, we observe a very good correspondence between chemical shifts of CA positions in the DNP-enhanced NCACX and the PDSD ${ }^{13} \mathrm{C}^{-13} \mathrm{C}$ spectra of the IF1-E30S complex at $100 \mathrm{~K}$ and an HNCA spectrum of free IF1 
acquired in solution state at room temperature, including the histidine and tyrosine residues discussed above (Figure S8). This suggests that when a ribosome binding factor is amenable to high resolution NMR investigation at room temperature (either in solution or in solid state), the assignment can be readily transferred to cryogenic temperatures for many residues, provided that this transition is not accompanied by large-scale conformational changes). In addition, the PDSD ${ }^{13} \mathrm{C}-{ }^{13} \mathrm{C}$ mixing $\left(\tau_{\mathrm{m}}=15 \mathrm{~ms}\right)$ after the transfer from ${ }^{15} \mathrm{~N}$ to ${ }^{13} \mathrm{C}$ in an NCACX experiment allows one to detect nitrogen-containing side chains (Egorova-Zachernyuk et al. 2001; Renault et al. 2012). As shown in Fig. 5, it is possible to unambiguously identify all five lysine side chains, and, based on chemical shift statistics of the BioMagResBank (Seavey et al. 1991), assign the signals at $\delta(\mathrm{N}) \sim 33 \mathrm{ppm}$ to $\mathrm{N}_{\zeta}$ lysine correlations. Overall, the resolution and linewidths observed in the NCACX spectrum suggest that high quality DNP-enhanced band-selective cross-polarization spectra can be acquired with frozen ribosome pellets. Furthermore, sequential labeling of unique pairs of amino acids $i$ and $i-1$ can facilitate sequential assignment of these spectra.
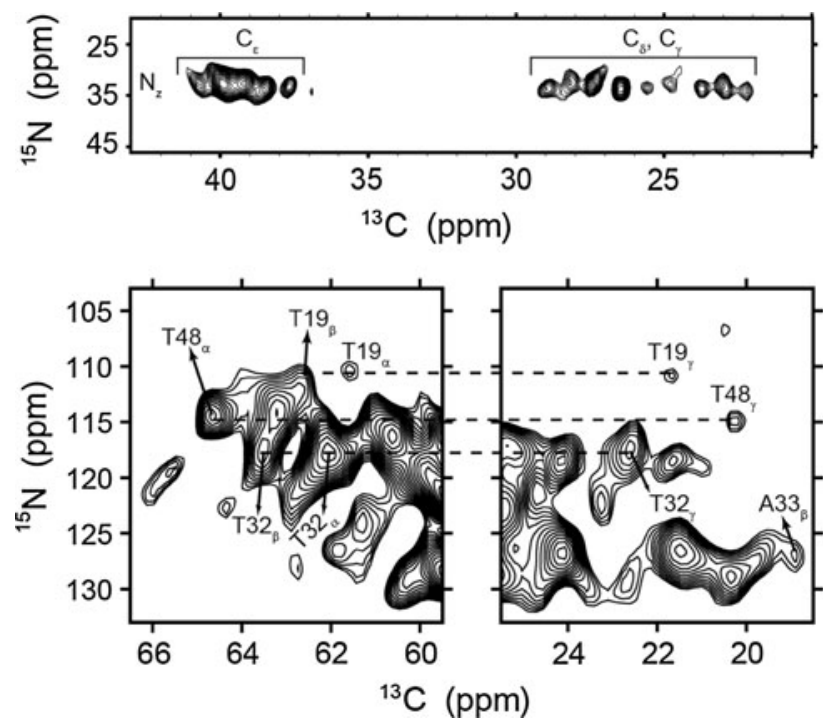

Fig. 5 Resolved regions of a ${ }^{15} \mathrm{~N}-{ }^{13} \mathrm{C}$ correlation spectrum (NCACX) of uniformly labeled IF1 bound to E30S (see Figure S3). After two cross-polarization (CP) steps from ${ }^{1} \mathrm{H}$ to ${ }^{15} \mathrm{~N}$ and from ${ }^{15} \mathrm{~N}$ to ${ }^{13} \mathrm{C},{ }^{13} \mathrm{C}-{ }^{13} \mathrm{C}$ exchange via PDSD $\left(\tau_{\text {mix }}=15 \mathrm{~ms}\right)$ allowed detecting two-bond $(\mathrm{N}-\mathrm{CB})$ and three-bond $(\mathrm{N}-\mathrm{CG})$ intra-residue correlations (lower panel) that facilitate the identification of the spin systems and their assignment. The highlighted connectivities of Thr residues and the methyl group of Ala33 can be readily distinguished. The tentative assignments are based on the CA chemical shifts in a solution-state HNCA spectrum of free IF1. Lysine residues (upper panel) and other nitrogen-containing side-chains that play a critical role in protein-protein interactions can be observed in the same spectrum and appear in their characteristic region

\section{Conclusions}

In conclusion, we have demonstrated that by direct pelleting of ribosome particles from glass-forming solvents it is possible to obtain significant DNP enhancements of ${ }^{13} \mathrm{C}$ and ${ }^{15} \mathrm{~N}$ signals and thus acquire two-dimensional correlation spectra from a few nanomoles of ribosomal complexes in a reasonable experimental timeframe. Since no solvent, apart from the amount contained in the pellet, is necessary, the sample density is high. The cryogenic temperatures in the vicinity of $100 \mathrm{~K}$ that are essential to achieve significant DNP enhancements greatly extend the lifetimes of samples that tend to degrade at room temperature, thus permitting extensive signal averaging. Furthermore, the improved Boltzmann distribution and reduced thermal noise lead to a higher signal-to-noise ratio. The frozen wet pellets can be readily prepared to study virtually any ribosomal complex without compromising spectral quality, as the ${ }^{13} \mathrm{C}$ linewidths are on the order of $1 \mathrm{ppm}$ or $100 \mathrm{~Hz}$ at $9.4 \mathrm{~T}$.

The experimental setting developed in this study thus demonstrates that when combined with selective labeling, DNP-MAS-NMR allows one to observe well-resolved signals from specific residues that are part of a ribosomal complex. The relationship between the chemical shift and linewidth of a resonance and the physical/chemical environment of the corresponding residue allows one to compare ribosomal complexes in different states to pinpoint, at the residue level, elements that experience changes in their environment. Given that is it possible to specifically label ribosomal ligands (as IF1 in this study), the complete ribosome or its individual elements including proteins or rRNA (Christodoulou et al. 2004; Llano-Sotelo et al. 2009; Erlacher et al. 2011) or the synthesized nascent chain (Hsu et al. 2007), DNP-MAS-NMR effectively extends our ability to study the structure of ribosome complexes that have, thus far, been beyond our reach. Although limited to the inspection of few residues (e.g. 5 in this study), this methodology can be, for example, applied to determine which residues of the polypeptide nascent chain interact with and/or change their conformation in response to the chaperones (e.g. TF, SecA, translocon) that guide the nascent chain in its acquisition of its final folded state and proper localization within the cell. Furthermore the ability to identify and assign spin systems (Fig. 5) implies the possibility to determine with relative certainty the conformation of specifically labelled residues. This would allow one to follow how the secondary structure of the polypeptide nascent chain evolves during its synthesis. Given the emerging evidence indicating a cross-talk between the nascent chain, the ribosome and the protein sorting and folding machinery, mainly mediated by structural signals imparted by the NC, we envisage that this methodology will play an important role to unravel this second code. 
Acknowledgments We are indebted to Paul Vasos, Martial Rey, Frank Engelke, Melanie Rosay, and Fabien Aussenac for their knowhow of instrumental aspects, to Christiane Aydin and Jutta Kreutz for ribosome preparations and Clemens Glaubitz for useful discussions and for the possibility to carry out initial experiments in his lab. This work was supported by grants from the Swiss National Science Foundation (SNSF) and the Swiss Commission for Technology and Innovation (CTI) to GB, and by grants from the Deutsche Forschungsgemeinschaft (FU579 1-3), the Cluster of Excellence 'Macromolecular complexes' at the Goethe University Frankfurt (DFG Project EXC 115), and the Human Frontiers of Science Program Young Investigators Award (HFSP67/07) to PF.

\section{References}

Abragam A (1961) The principles of nuclear magnetism. Clarendon Press, Oxford

Abragam A, Goldman M (1978) Principles of dynamic nuclearpolarization. Rep Prog Phys 41(3):395-467

Ader C, Frey S, Maas W, Schmidt HB, Gorlich D, Baldus M (2010) Amyloid-like interactions within nucleoporin FG hydrogels. Proc Natl Acad Sci USA 107(14):6281-6285

Amand B, Pochon F, Lavalette D (1977) Rotational diffusion of Escherichia coli ribosomes. I.-Free $70 \mathrm{~S}, 50 \mathrm{~S}$ and $30 \mathrm{~S}$ particles. Biochimie 59(10):779-784

Angell CA (2002) Liquid fragility and the glass transition in water and aqueous solutions. Chem Rev 102(8):2627-2650

Bajaj VS, Mak-Jurkauskas ML, Belenky M, Herzfeld J, Griffin RG (2009) Functional and shunt states of bacteriorhodopsin resolved by $250 \mathrm{GHz}$ dynamic nuclear polarization-enhanced solid-state NMR. Proc Natl Acad Sci USA 106(23):9244-9249

Baldus M, Petkova AT, Herzfeld J, Griffin RG (1998) Cross polarization in the tilted frame: assignment and spectral simplification in heteronuclear spin systems. Mol Phys 95(6):1197-1207

Barnes AB, Paepe GD, van der Wel PC, Hu KN, Joo CG, Bajaj VS, Mak-Jurkauskas ML, Sirigiri JR, Herzfeld J, Temkin RJ, Griffin RG (2008) High-field dynamic nuclear polarization for solid and solution biological NMR. Appl Magn Reson 34(3-4):237-263

Barnes AB, Corzilius B, Mak-Jurkauskas ML, Andreas LB, Bajaj VS, Matsuki Y, Belenky ML, Lugtenburg J, Sirigiri JR, Temkin RJ, Herzfeld J, Griffin RG (2010) Resolution and polarization distribution in cryogenic DNP/MAS experiments. Phys Chem Chem Phys 12(22):5861-5867

Bayro MJ, Debelouchina GT, Eddy MT, Birkett NR, MacPhee CE, Rosay M, Maas WE, Dobson CM, Griffin RG (2011) Intermolecular structure determination of amyloid fibrils with magicangle spinning and dynamic nuclear polarization NMR. J Am Chem Soc 133(35):13967-13974

Bertini I, Luchinat C, Parigi G, Ravera E, Reif B, Turano P (2011) Solid-state NMR of proteins sedimented by ultracentrifugation. Proc Natl Acad Sci USA 108(26):10396-10399

Bertini I, Engelke F, Gonnelli L, Knott B, Luchinat C, Osen D, Ravera E (2012a) On the use of ultracentrifugal devices for sedimented solute NMR. J Biomol NMR 54(2):123-127

Bertini I, Engelke F, Luchinat C, Parigi G, Ravera E, Rosa C, Turano P (2012b) NMR properties of sedimented solutes. Phys Chem Chem Phys 14(2):439-447

Cabrita LD, Hsu ST, Launay H, Dobson CM, Christodoulou J (2009) Probing ribosome-nascent chain complexes produced in vivo by NMR spectroscopy. Proc Natl Acad Sci USA 106(52):22239-22244

Cady SD, Schmidt-Rohr K, Wang J, Soto CS, Degrado WF, Hong M (2010) Structure of the amantadine binding site of influenza M2 proton channels in lipid bilayers. Nature 463(7281):689-692
Carter AP, Clemons WM, Brodersen DE, Morgan-Warren RJ, Hartsch T, Wimberly BT, Ramakrishnan V (2001) Crystal structure of an initiation factor bound to the $30 \mathrm{~S}$ ribosomal subunit. Science 291(5503):498-501

Celano B, Pawlik RT, Gualerzi CO (1988) Interaction of Escherichia coli translation-initiation factor IF-1 with ribosomes. Eur J Biochem 178(2):351-355

Christodoulou J, Larsson G, Fucini P, Connell SR, Pertinhez TA, Hanson CL, Redfield C, Nierhaus KH, Robinson CV, Schleucher J, Dobson CM (2004) Heteronuclear NMR investigations of dynamic regions of intact Escherichia coli ribosomes. Proc Natl Acad Sci USA 101(30):10949-10954

Debelouchina GT, Bayro MJ, van der Wel PC, Caporini MA, Barnes AB, Rosay M, Maas WE, Griffin RG (2010) Dynamic nuclear polarization-enhanced solid-state NMR spectroscopy of GNNQQNY nanocrystals and amyloid fibrils. Phys Chem Chem Phys 12(22):5911-5919

Egorova-Zachernyuk TA, Hollander J, Fraser N, Gast P, Hoff AJ, Cogdell R, de Groot HJ, Baldus M (2001) Heteronuclear 2Dcorrelations in a uniformly $[13 \mathrm{C}, 15 \mathrm{~N}]$ labelled membraneprotein complex at ultra-high magnetic fields. J Biomol NMR 19(3):243-253

Eichmann C, Preissler S, Riek R, Deuerling E (2010) Cotranslational structure acquisition of nascent polypeptides monitored by NMR spectroscopy. Proc Natl Acad Sci USA 107(20):91119116

Erlacher MD, Chirkova A, Voegele P, Polaceck N (2011) Generation of chemically engineered ribosomes for mutagenesis studies on protein biosynthesis. Nat Protoc 6:580-592

Fedyukina DV, Cavagnero S (2011) Protein folding at the exit tunnel. Annu Rev Biophys 40:337-359

Gardiennet C, Schutz AK, Hunkeler A, Kunert B, Terradot L, Bockmann A, Meier BH (2012) Sedimented sample of a $59 \mathrm{kDa}$ dodecameric helicase yields high-resolution solid-state NMR spectra. Angew Chem Int Ed Engl 51(31):7855-7858

Georgieva ER, Roy AS, Grigoryants VM, Borbat PP, Earle KA, Scholes CP, Freed JH (2012) Effect of freezing conditions on distances and their distributions derived from double electron electron resonance (DEER): a study of doubly-spin-labeled T4 lysozyme. J Magn Reson 216:69-77

Hall DA, Maus DC, Gerfen GJ, Inati SJ, Becerra LR, Dahlquist FW, Griffin RG (1997) Polarization-enhanced NMR spectroscopy of biomolecules in frozen solution. Science 276(5314):930-932

Helgstrand M, Mandava CS, Mulder FA, Liljas A, Sanyal S, Akke M (2007) The ribosomal stalk binds to translation factors IF2, EF$\mathrm{Tu}, \mathrm{EF}-\mathrm{G}$ and RF3 via a conserved region of the L12 C-terminal domain. J Mol Biol 365:468-479

Hiller M, Krabben L, Vinothkumar KR, Castellani F, van Rossum BJ, Kuhlbrandt W, Oschkinat H (2005) Solid-state magic-angle spinning NMR of outer-membrane protein G from Escherichia coli. ChemBioChem 6(9):1679-1684

Hsu ST, Fucini P, Cabrita LD, Launay H, Dobson CM, Christodoulou J (2007) Structure and dynamics of a ribosome-bound nascent chain by NMR spectroscopy. Proc Natl Acad Sci USA 104(42):16516-16521

Hsu STD, Cabrita LD, Fucini P, Christodoulou J, Dobson CM (2009) Probing side-chain dynamics of a ribosome-bound nascent chain using methyl NMR spectroscopy. J Am Chem Soc 131(24): 8366-8367

Kiesewetter MK, Corzilius B, Smith AA, Griffin RG, Swager TM (2012) Dynamic nuclear polarization with a water-soluble rigid biradical. J Am Chem Soc. doi:10.1021/ja212054e

Lange S, Linden AH, Akbey U, Franks WT, Loening NM, van Rossum BJ, Oschkinat H (2012) The effect of biradical concentration on the performance of DNP-MAS-NMR. J Magn Reson 216:209-212 
Lelli M, Gajan D, Lesage A, Caporini MA, Vitzthum V, Mieville P, Heroguel F, Rascon F, Roussey A, Thieuleux C, Boualleg M, Veyre L, Bodenhausen G, Coperet C, Emsley L (2011) Fast characterization of functionalized silica materials by silicon-29 surface-enhanced NMR spectroscopy using dynamic nuclear polarization. J Am Chem Soc 133(7):2104-2107

Linden AH, Franks WT, Akbey U, Lange S, van Rossum BJ, Oschkinat H (2011) Cryogenic temperature effects and resolution upon slow cooling of protein preparations in solid state NMR. J Biomol NMR 51(3):283-292

Llano-Sotelo B, Hickerson RP, Lancaster L, Noller HF, Mankin AS (2009) Fluorescently labelled ribosomes as a tool for analyzing antibiotic binding. RNA 15(8):1597-1604

Martin RW, Zilm KW (2003) Preparation of protein nanocrystals and their characterization by solid state NMR. J Magn Reson 165(1):162-174

Mulder FA, Bouakaz L, Lundell A, Venkataramana M, Liljas A, Akke M, Sanyal S (2004) Conformation and dynamics of ribosomal stalk protein L12 in solution and on the ribosome. Biochemistry 43(20):5930-5936

Noeske J, Cate JH (2012) Structural basis for protein synthesis: snapshots of the ribosome in motion. Curr Opin Struct Biol 22(6):743-749

Pauli J, Baldus M, van Rossum B, de Groot H, Oschkinat H (2001) Backbone and side-chain $\mathrm{C}-13$ and $\mathrm{N}-15$ signal assignments of the alpha-spectrin $\mathrm{SH} 3$ domain by magic angle spinning solidstate NMR at 17.6 Tesla. ChemBioChem 2(4):272-281

Reggie L, Lopez JJ, Collinson I, Glaubitz C, Lorch M (2011) Dynamic nuclear polarization-enhanced solid-state NMR of a 13C-labeled signal peptide bound to lipid-reconstituted Sec translocon. J Am Chem Soc 133(47):19084-19086

Renault M, Pawsey S, Bos MP, Koers EJ, Nand D, Tommassen-van Boxtel R, Rosay M, Tommassen J, Maas WE, Baldus M (2012) Solid-state NMR spectroscopy on cellular preparations enhanced by dynamic nuclear polarization. Angew Chem Int Ed Engl 51(12):2998-3001

Rutkowska A, Beerbaum M, Rajagopalan N, Fiaux J, Schmieder P, Kramer G, Oschkinat H, Bukau B (2009) Large-scale purification of ribosome-nascent chain complexes for biochemical and structural studies. FEBS Lett 583(14):2407-2413

Schmeing TM, Ramakrishnan V (2009) What recent ribosome structures have revealed about the mechanism of translation. Nature 461(7268):1234-1242

Seavey BR, Farr EA, Westler WM, Markley JL (1991) A relational database for sequence-specific protein NMR data. J Biomol NMR 1(3):217-236
Sette M, van Tilborg P, Spurio R, Kaptein R, Paci M, Gualerzi CO, Boelens R (1997) The structure of the translational initiation factor IF1 from $E$. coli contains an oligomer-binding motif. EMBO J 16(6):1436-1443

Shewmaker F, Wickner RB, Tycko R (2006) Amyloid of the prion domain of Sup35p has an in-register parallel beta-sheet structure. Proc Natl Acad Sci USA 103(52):19754-19759

Song C, Hu KN, Joo CG, Swager TM, Griffin RG (2006) TOTAPOL: a biradical polarizing agent for dynamic nuclear polarization experiments in aqueous media. J Am Chem Soc 128(35):1138511390

van der Wel PC, Hu KN, Lewandowski J, Griffin RG (2006) Dynamic nuclear polarization of amyloidogenic peptide nanocrystals: GNNQQNY, a core segment of the yeast prion protein Sup35p. J Am Chem Soc 128(33):10840-10846

Vitzthum V, Borcard F, Jannin S, Morin M, Mieville P, Caporini MA, Sienkiewicz A, Gerber-Lemaire S, Bodenhausen G (2011) Fractional spin-labelling of polymers for enhancing NMR sensitivity by solvent-free dynamic nuclear polarization. Chemphyschem 12(16):2929-2932

Vitzthum V, Mieville P, Carnevale D, Caporini MA, Gajan D, Coperet C, Lelli M, Zagdoun A, Rossini AJ, Lesage A, Emsley L, Bodenhausen G (2012) Dynamic nuclear polarization of quadrupolar nuclei using cross polarization from protons: surface-enhanced aluminium-27 NMR. Chem Commun 48(14): 1988-1990

Wasmer C, Lange A, Van Melckebeke H, Siemer AB, Riek R, Meier BH (2008) Amyloid fibrils of the HET-s(218-289) prion form a beta solenoid with a triangular hydrophobic core. Science 319(5869):1523-1526

Wilson DN (2009) The A-Z of bacterial translation inhibitors. Crit Rev Biochem Mol Biol 44(6):393-433

Zagdoun A, Rossini AJ, Gajan D, Bourdolle A, Ouari O, Rosay M, Maas WE, Tordo P, Lelli M, Emsley L, Lesage A, Coperet C (2012) Non-aqueous solvents for DNP surface enhanced NMR spectroscopy. Chem Commun (Camb) 48(5):654-656 